\title{
Unfavorable pathological characteristics in familial colorectal cancer with low-level microsatellite instability
}

\author{
Carolien M Kets ${ }^{1}$, Nicoline Hoogerbrugge ${ }^{1}$, Danielle Bodmer ${ }^{1}$, Riki Willems ${ }^{2}$, \\ Han G Brunner ${ }^{1}$, Joannes HJM van Krieken ${ }^{2}$ and Marjolijn JL Ligtenberg ${ }^{1,2}$ \\ ${ }^{1}$ Department of Human Genetics, Radboud University Nijmegen Medical Centre, Nijmegen, The Netherlands \\ and ${ }^{2}$ Department of Pathology, Radboud University Nijmegen Medical Centre, Nijmegen, The Netherlands
}

\begin{abstract}
A high degree of microsatellite instability (MSI) in colorectal cancer (CRC) is a hallmark of hereditary nonpolyposis colorectal cancer (HNPCC), caused by germline defects in the mismatch repair (MMR) genes. A low degree of instability (less than $30 \%$ of the microsatellites) is seen in a subset of tumors. To clarify the significance of this low degree of MSI phenotype, we studied the differences between patients with colorectal tumors with high-level, low-level and no MSI. Colorectal tumors with no $(n=68)$ and low-level $(n=18)$ MSI of patients clinically suspected of HNPCC were compared to colorectal tumors with high-level MSI $(n=12)$ of patients that carry a pathogenic germline mutation in a MMR gene. Compared to tumors with no MSI, tumors with low-level MSI were classified more frequently as stage T3 or T4 $(100 \%$ vs $68 \%$ respectively), and showed less immune response $(P=0.02)$. No significant differences in familial CRC risk were found by comparing pedigrees of these two groups of tumors. Compared to the group of tumors with high-level MSI, the group of tumors with low-level MSI had a less suspicious family history, a higher percentage of lymph node metastasis (56 vs 17\%), and less immune response. Thus, with respect to genetic risks, familial CRC can be divided into two groups: Tumors with high-level MSI and tumors with low-level or no MSI. However, tumors with low-level MSI show unfavorable pathological characteristics compared to tumors with no and tumors with high-level MSI. These differences suggest a distinct underlying biology of CRC with low-level MSI.
\end{abstract}

Modern Pathology (2006) 19, 1624-1630. doi:10.1038/modpathol.3800701; published online 15 September 2006

Keywords: HNPCC; low-level microsatellite instability; morphology; prognosis; familial colorectal cancer

Hereditary non-polyposis colorectal cancer (HNPCC) is caused by a defect in one of the mismatch repair (MMR) genes (eg MLH1, MSH2, MSH6 and PMS2). Failure of the DNA MMR system causes microsatellite instability (MSI) in tumors of HNPCC patients. MSI analysis is used to select patients for germline mutation analysis of the MMR genes. When only a small number of the tested markers shows instability (less than $30 \%$ ) the tumor is considered MSI low. ${ }^{1}$ In clinical practice both the MSI low group and the microsatellite stable (MSS) group are considered as being not suspicious of HNPCC. However, the significance of MSI low is not well understood. Although the existence of a defined class of MSI low tumors is questioned, ${ }^{2}$ there are studies that show molecular differences

Correspondence: DR CM Kets, MD, Department of Human Genetics, Radboud University Nijmegen Medical Centre, Code 849 Clinical Genetics, PO Box 9101, Nijmegen 6500 HB, The Netherlands.

E-mail: m.kets@antrg.umcn.nl

Received 6 June 2006; revised 18 August 2006; accepted 21 August 2006; published online 15 September 2006 between MSI low and MSS tumors, for example, a higher KRAS mutation rate, ${ }^{3-5}$ less frequent loss of heterozygosity of $5 \mathrm{q},{ }^{4}$ a higher rate of $M G M T$ promoter hypermethylation ${ }^{5}$ and distinct gene expression profiles in microarray experiments. ${ }^{6}$ Moreover, MSI low tumors may confer a worse prognosis. ${ }^{7,8}$ Little is known about the histopathological features of familial MSI low tumors. To clarify the importance of MSI low status in colorectal tumors of patients suspected of HNPCC, tumors were classified as MSI low or MSS according to strict criteria. Subsequently, the different groups were compared with regard to family history, clinicopathological and molecular features. In this study, MSI high colorectal tumors of MMR mutation carriers were used as reference.

\section{Materials and methods}

Patient Selection and Family History

In total, 177 colorectal cancer (CRC) patients clinically suspected of HNPCC, attending our 
hereditary cancer clinic between July 2002 and September 2004, were studied. In these families MSI analysis was performed in the tumor of the youngest patient available. When MSI analysis was performed in more than one tumor within a family, only the tumor of the youngest family member was included in the analysis of the family history. The family history was studied for fulfillment of Amsterdam criteria ${ }^{9}$ and modified Bethesda guidelines $2001 .{ }^{10}$ Inheritance patterns were divided in (1) autosomal dominant (AD), defined as first-degree relatives with CRC in two or more generations, (2) $\mathrm{AD}$ with reduced penetrance defined as two or more nonfirst-degree relatives with CRC and (3) autosomal recessive (AR) which is defined as two or more first-degree relatives with CRC in one generation. The study was performed according to the rules of the Medical Ethics Committee of the Radboud University Nijmegen Medical Centre.

\section{Molecular Testing}

MSI analysis was performed with the Bethesda microsatellite panel D2S123, D5S346, D17S250, $B A T 25, B A T 26^{1}$ using methods described previously. ${ }^{11}$ Normal and tumor tissues were extracted from formalin-fixed and paraffin-embedded tissues. MSI in MSI low tumors is often confined to the addition of a single dinucleotide repeat (Figure 1a). If the alleles of a given marker differ only one repeat, the pattern of the addition of a single dinucleotide repeat to the smaller allele cannot be discriminated from that of loss of heterozygosity of the smaller allele (Figure 1c). Therefore, these markers were
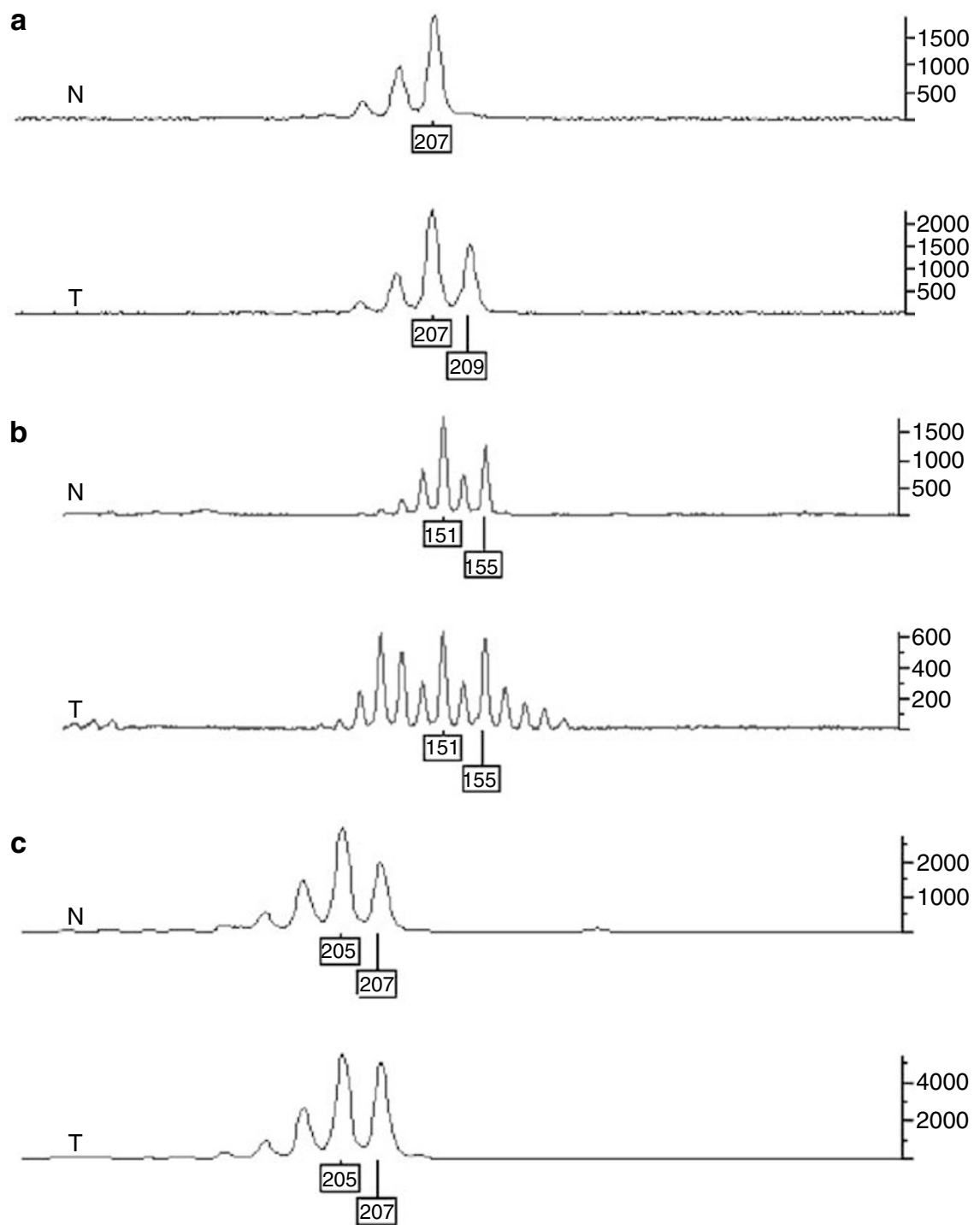

Figure 1 Genescan patterns of microsatellite markers of three pairs of normal (N) and corresponding tumor (T) tissue. (a) Example of a characteristic MSI pattern in an MSI low tumor with addition of only one dinucleotide repeat. (b) Example of an obvious MSI pattern. (c) Example of an unclear instability pattern; increase in allele 207 can be caused by an addition of 1 dinucleotide to or loss of allele 205. 
scored as nonconclusive. A tumor was regarded as MSS when all five markers were classified as stable. A tumor was considered MSI low when one dinucleotide marker showed MSI. In such a case, five extra markers were tested. Extending the number of markers never resulted in reclassification of these tumors as MSI high. Only MSI high tumors of patients with a germline mutation in one of the MMR genes were used in this study. The pathogenic germline mutations in the MSI high group consisted of six MLH1 mutations, five MSH2 mutations and one MSH6 mutation. In one deceased patient with an MSH2 mutation the germline mutation was concluded from mutation analysis of affected family members. All MSI high tumors showed MSI in at least three of the standard markers.

Of the 177 CRCs, 93 could be classified unambiguously using these criteria. 84 tumors were excluded; 69 non-MSI high tumors because one or more markers were not interpretable and 15 MSI high tumors were excluded because a MMR mutation was not found at that moment. The MSI low group was enlarged with all five tumors that could be unambiguously classified as MSI low tumors from the subset of tumors of HNPCC suspected patients tested between 1998 and June 2002. A total of 98 CRC tumors (18 MSI low; 68 MSS and 12 MSI high) were included for further analysis.

Immunohistochemistry (IHC) of MLH1, PMS2, MSH2 and MSH6 was performed on 53 available tissue blocks, including 10 MSI low tumors, 36 MSS tumors and seven MSI high tumors using monoclonal antibodies against MLH1 (Pharmingen code: 51-1327gr), PMS2 (Pharmingen code: 556415), MSH2 (Oncogene code: NA26) and MSH6 (Transduction lab code: G70220).

\section{Clinicopathological Features}

Data relating to cancer site, depth of invasion and nodal spread were obtained from pathological records, using the current (2002) TNM staging system. Right-sided colon cancer was defined as located proximal to the splenic flexure. Morphology is reviewed by a single experienced pathologist (JHJMvK) using hematoxylin and eosin (H\&E) sections. The following additional pathological parameters were scored:

(1) Tumor differentiation: well, moderate or poor, according to WHO criteria, ${ }^{12}$ (2) growth pattern: infiltrative partly circumscribed $(<50 \%)$ or extensively circumscribed ( $>50 \%$ ), (3) Crohn's-like lymphoid reaction: discrete lymphoid aggregates at the outside of the muscularis propria; no, moderate or extensive, using criteria defined by Graham et al, ${ }^{13}$ (4) mucinous differentiation: none $(0 \%)$, moderate $(<50 \%)$ or extensive $(>50 \%)$, (5) lymphangioinvasive growth: none, focal or extensive, (6) amount of individual lymphocytes in the tumor: presence of intraepithelial lymphocytes; few, intermediate or many, as described by van den Bos et al, ${ }^{14}$ (7) amount of peritumoral lymphocytes: cap or mantle of lymphocytes surrounding the invasive front; no, partially or complete, (8) amount of intertumoral lymphocytes: lymphocytes between tumor glands; few, intermediate or many.

To compare MSS, MSI high and MSI low tumors the pathological parameters are subdivided in two categories. For tumor differentiation, a subdivision is made in well differentiation and moderate to poor differentiation. For Crohn's-like lymphoid reaction, lymphangioinvasive growth, intraepithelial lymphocytes, peritumoral lymphocytes and intertumoral lymphocytes, the scores are divided in the presence or absence. For mucinous differentiation only the score extensive $(>50 \%)$ is counted as presence, thus representing mucinous carcinoma.

\section{Statistical Analysis}

Patient and family characteristics

Independent sample T-tests and one way ANOVA's with Bonferroni correction for multiple testing were used to compare mean values of continuous variables in different groups. Two-group-comparisons of dichotomous variables were performed with Fisher exact tests only for variables for which logistic regression revealed significant differences between the three groups.

\section{Clinicopathological features}

Comparisons of MSI low and MSS tumors on dichotomous variables were performed with Fisher exact tests. For the four items reflecting immune response (Crohn's-like lymphoid reaction, intraepithelial, peritumoral and intertumoral lymphocytes) multiple analysis by generalized estimation equation (GEE) was performed to compare the MSI low group and the MSS group.

$P$-values $<0.05$ are considered to indicate significance. For GEE analysis we used SAS version 8.0, all other analyses were performed using SPSS, version 12.0.

\section{Results}

\section{Patient and Family Characteristics}

In five families, two family members were included; in two families one patient had an MSI low tumor and the other an MSS tumor, in three families both patients had an MSS tumor. For pedigree analysis only the youngest tested patient was included. The mean age of CRC diagnosis was 48 years (range 23-72 years) with 56 males (60\%). The mean age of the youngest relative in the family with CRC was 45 years (range 23-70) and the mean age of CRC diagnosis of all relatives with CRC was 53 years (range 23-74). A family history of CRC was present in 79 patients $(85 \%)$. In 32 of these families the 
Amsterdam criteria were fulfilled. In Table 1 and 2, the characteristics are described for the MSI low, MSS and MSI high groups separately.

There were no significant differences between the MSI low and MSS group in fulfilling Amsterdam criteria and Bethesda guidelines. Although a second HNPCC-related tumor (Bethesda guideline A) and age at diagnosis below 50 years (Bethesda guideline C) occurred more often in the MSI low group as compared to the MSS group, these differences did not reach statistical significance.
In all groups an $\mathrm{AD}$ inheritance pattern was most frequent. The inheritance patterns of the MSI low group and MSS group are comparable. Both MSI low and MSS groups had a less suspicious family history as compared to the MSI high group, fulfilling the Amsterdam I and II criteria significantly less often. Additionally, the mean age at diagnosis of all relatives with CRC and of the mean age of the youngest relative with CRC in the family is significantly higher than in the MSI high group.

Table 1 Patient and family characteristics

\begin{tabular}{|c|c|c|c|c|c|c|c|c|c|c|}
\hline & \multicolumn{2}{|c|}{$\begin{array}{l}\text { MSI low } \\
(\mathrm{n}=16)\end{array}$} & \multicolumn{2}{|c|}{$\begin{array}{c}M S S \\
(\mathrm{n}=65)\end{array}$} & \multicolumn{2}{|c|}{$\begin{array}{l}\text { MSI high } \\
(\mathrm{n}=12)\end{array}$} & \multirow[t]{2}{*}{$\begin{array}{l}\mathrm{P} \text {-value logistic } \\
\text { regression Wald test }\end{array}$} & \multicolumn{3}{|c|}{$\begin{array}{c}\text { P-values for pair wise comparison } \\
\text { Fisher exact }\end{array}$} \\
\hline & No. & $\%$ & No. & $\%$ & No. & $\%$ & & $\begin{array}{l}\text { MSI low } \\
\text { vs MSS }\end{array}$ & $\begin{array}{c}\text { MSI low } \\
\text { vs MSI high }\end{array}$ & $\begin{array}{l}\text { Non-MSI high } \\
\text { vs MSI high }\end{array}$ \\
\hline Male & 12 & 75 & 38 & 58 & 6 & 50 & 0.368 & & & 0.532 \\
\hline Amsterdam I+ ${ }^{\mathrm{a}}$ & 3 & 19 & 13 & 20 & 8 & 67 & 0.008 & 1.000 & 0.019 & 0.002 \\
\hline Amsterdam II+ ${ }^{\mathrm{a}}$ & 4 & 25 & 18 & 28 & 10 & 83 & 0.006 & 1.000 & 0.006 & $<\mathbf{0 . 0 0 1}$ \\
\hline Bethesda $\mathrm{A}+{ }^{\mathrm{a}}$ & 4 & 25 & 10 & 15 & 4 & 33 & 0.303 & & & 0.238 \\
\hline Bethesda B+a & 8 & 50 & 31 & 48 & 10 & 83 & 0.111 & & & 0.030 \\
\hline Bethesda C+ ${ }^{\mathrm{a}}$ & 11 & 69 & 39 & 60 & 9 & 75 & 0.550 & & & 0.525 \\
\hline Familial CRC & 14 & 88 & 53 & 82 & 12 & 100 & 0.854 & & & 0.202 \\
\hline Inheritance & \multicolumn{2}{|c|}{$\mathrm{n}=14$} & \multicolumn{2}{|c|}{$\mathrm{n}=53$} & \multicolumn{2}{|c|}{$\mathrm{n}=12$} & & & & \\
\hline $\mathrm{AD}^{\mathrm{b}}$ & 7 & 50 & 24 & 45 & 8 & 67 & 0.421 & & & 0.225 \\
\hline $\begin{array}{l}\text { AD red. } \\
\text { penetrance }^{\mathrm{b}}\end{array}$ & 6 & 43 & 25 & 47 & 4 & 33 & 0.683 & & & 0.533 \\
\hline $\mathrm{AR}^{\mathrm{b}}$ & 1 & 7 & 4 & 8 & 0 & 0 & 0.999 & & & 1.000 \\
\hline
\end{tabular}

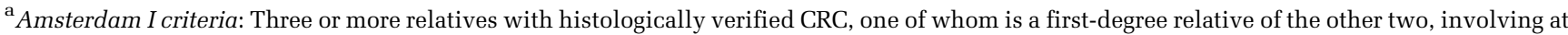
least two generations and one or more cancer cases diagnosed before the age of 50 years (FAP should be excluded). Amsterdam II criteria: Three or more relatives with an HNPCC-associated cancer (CRC, cancer of the endometrium, gastric carcinoma, ovarian carcinoma, cancer of small bowel, brain, ureter, renal pelvis, carcinoma of the brain, hepatobiliary tract or sebaceous tumors), one of whom is a first-degree relative of the other two, involving at least two generations and one or more cancer cases diagnosed before the age of 50 years (FAP should be excluded and tumors should be verified whenever possible). Bethesda A: Individuals with two HNPCC-related cancers, including synchronous and metachronous CRCs or endometrial, ovarian, gastric, hepatobiliary, small bowel cancer and transitional cell carcinoma of the renal pelvis or ureter. Bethesda B: Individuals with CRC and a first-degree relative with CRC and/or HNPCC-related extracolonic cancer and/or a colorectal adenoma; one of the cancers diagnosed at age $<50$ years, and the adenoma diagnosed at age $<40$ years. Bethesda C: Individuals with CRC or endometrial cancer diagnosed at age $<50$ years.

${ }^{\mathrm{b}}$ Inheritance patterns: AD: autosomal dominant; First-degree relatives with CRC in two or more generations. AD red. penetrance: autosomal dominant with reduced penetrance; CRC in two or more relatives, not all relatives are first-degree relatives. AR: autosomal recessive; two or more first-degree relatives with CRC in one generation.

Bold $P$-values: $P$-values $<0.05$.

Table 2 Age at diagnosis of patients and relatives

\begin{tabular}{|c|c|c|c|c|c|c|c|c|c|c|}
\hline & \multicolumn{2}{|c|}{$\begin{array}{c}\text { MSI low } \\
(\mathrm{n}=16)\end{array}$} & \multicolumn{2}{|c|}{$\begin{array}{c}M S S \\
(\mathrm{n}=65)\end{array}$} & \multicolumn{2}{|c|}{$\begin{array}{c}\text { MSI high } \\
(\mathrm{n}=12)\end{array}$} & \multicolumn{3}{|c|}{$\begin{array}{c}\mathrm{P} \text {-values } 1 \text {-way ANOVA } \\
\text { \{mean difference in years\} } \\
\text { [95\% confidence interval] }\end{array}$} & \multirow{2}{*}{$\begin{array}{c}\text { Independent } \\
\text { sample T-test } \\
\begin{array}{c}\text { Non-MSI high } \\
\text { vs MSI high }\end{array}\end{array}$} \\
\hline & Years & Range & Years & Range & Years & Range & $\begin{array}{c}\text { Comparison } \\
\text { MSS, MSI low, } \\
\text { MSI high }\end{array}$ & $\begin{array}{c}\text { MSI low } \\
\text { vs MSS }\end{array}$ & $\begin{array}{c}\text { MSI low } \\
\text { vs MSI high }\end{array}$ & \\
\hline Mean age at diagnosis & 49 & $33-71$ & 49 & $23-71$ & 45 & $27-72$ & 0.537 & & & 0.264 \\
\hline $\begin{array}{l}\text { Mean age at diagnosis } \\
\text { all family members } \\
\text { with CRC }\end{array}$ & 53 & $33-74$ & 54 & $23-72$ & 47 & $37-56$ & 0.128 & & & $\begin{array}{c}\mathbf{0 . 0 4 5} \\
(6.8 \text { years }) \\
(0.2-13.4)\end{array}$ \\
\hline $\begin{array}{l}\text { Mean age at diagnosis } \\
\text { youngest family } \\
\text { member with CRC }\end{array}$ & 46 & $33-64$ & 46 & $23-70$ & 37 & $27-54$ & 0.016 & 1.000 & $\begin{array}{c}\mathbf{0 . 0 4} \\
(9.3 \text { years }) \\
(0.3-18.2)\end{array}$ & $\begin{array}{c}\mathbf{0 . 0 0 4} \\
(8.7 \text { years }) \\
(2.8-14.5)\end{array}$ \\
\hline
\end{tabular}

Bold $P$-values: $P$-values $<0.05$. 


\section{Clinicopathological Features}

Thirty $(31 \%)$ tumors were localized at the right of the colon. The minority was well differentiated $(11 \%)$ with $33 \%$ showing a circumscribed growth pattern. No differences were found between the MSI low and MSS groups in mucinous differentiation grade and growth pattern. The MSI low tumors showed a lower amount of tumor infiltrating, peritumoral and intertumoral lymphocytes $(P=0.043)$ as compared to MSS tumors (Table 3, Figure 2). GEE analysis showed a significantly lower antitumor immune response, reflected by the items tumor infiltrating, peritumoral and intertumoral lymphocytes and Crohn's-like lymphoid reaction, of MSI low tumors compared to MSS tumors $(P=0.02)$. The MSI low tumors tended to have positive lymph nodes more often (Figure 3 ) and had a higher T-grade $(P=0.004)$.

Both MSI low and MSS tumors were better differentiated, less often mucinous, showed less lymphocytic reaction and had more often lymph node metastasis compared to MSI high tumors.

\section{Molecular Features}

In the MSI low group, the dinucleotide repeat marker D5S346 showed instability in 6\%,D17S250 in $39 \%$ and $D 2 S 123$ in $56 \%$ of tumors. In the MSI high group each dinucleotide marker showed instability in $90-92 \%$ of tumors. The nature of the instability pattern is different between the MSI low and MSI high group (Figure 1a and b). Addition of only one dinucleotide repeat is seen in $78 \%$ of instable dinucleotide repeat markers in the MSI low group, but in only $13 \%$ of those in MSI high tumors (Figure 1a).

Thirty-six MSS and 10 MSI low tumors were available for immunohistochemical staining: no loss of staining of MLH1, PMS2, MSH2 or MSH6 was found. In some cases weak or heterogeneous staining of the proteins was seen, most frequently of
MLH1 and MSH6 proteins. To investigate whether (partial) hypermethylation of the MLH1 promoter could explain the MLH1 staining variability, additional methylation analyses of the $M L H 1$ promoter was performed in two MSI low and five MSS tumors. No hypermethylation of the MLH1 promoter was found. This was also the case in three MSI low and one MSS tumors with strong staining of the MLH1 protein (data not shown). We conclude that the staining variability was not due to MMR gene defects. In the seven available MSI high tumors the immunohistochemical pattern was in concordance with the germline mutations.

\section{Discussion}

In our study of familial CRC, MSI low tumors more frequently have a high T-grade, lymph node metastasis and lymphangioinvasive growth, as compared to MSS tumors. Additionally, MSI low tumors show a lower antitumor immune response (lack of intraepithelial, peritumoral and intertumoral lymphocytes and Crohn's-like lymphoid reaction), a known marker for worse prognosis. ${ }^{15,16}$ These data may explain the worse prognosis of MSI low tumors that was shown in the studies of Kohonen-Corish et $a l^{7}$ and Wright et $a l^{8}{ }^{8}$ Data on survival are not available in our study. In contrast to previous studies on sporadic tumors, ${ }^{8,17,18}$ we found differences in clinicopathological features between MSI low and MSS tumors. This may be explained by the fact that we focused on familial CRCs, which might generate groups with more homogeneous etiologies.

The family history in the MSI low group is comparable to that in the MSS group. Individual patient characteristics that might be indicative for a genetic susceptibility, like a synchronous or metachronous tumor development and CRC at young age occur slightly more often in the MSI low group than in the MSS group. The fact that the MSI low group has a less suspicious family history than the MSI

Table 3 Tumor characteristics

\begin{tabular}{|c|c|c|c|c|c|c|c|}
\hline & \multicolumn{2}{|c|}{ MSI low } & \multicolumn{2}{|c|}{ MSS } & \multirow{2}{*}{$\begin{array}{c}\text { P-value MSI low vs MSS } \\
\text { Fisher exact }\end{array}$} & \multicolumn{2}{|c|}{ MSI high } \\
\hline & No. & $\%$ & No. & $\%$ & & No. & $\%$ \\
\hline Right tumor localization & $4 / 18$ & 22 & $20 / 68$ & 29 & 0.769 & $6 / 12$ & 50 \\
\hline Well differentiated & $2 / 18$ & 11 & $9 / 62$ & 15 & 1.000 & $0 / 12$ & 0 \\
\hline Mucinous differentiation & $2 / 18$ & 11 & $8 / 59$ & 14 & 1.000 & $3 / 12$ & 25 \\
\hline Circumscribed growth pattern & $7 / 18$ & 39 & $20 / 59$ & 34 & 0.780 & $5 / 12$ & 42 \\
\hline Crohn's-like lymphoid reaction & $10 / 18$ & 56 & $33 / 54$ & 61 & 0.783 & $6 / 12$ & 50 \\
\hline Intraepithelial lymphocytes & $0 / 18$ & 0 & $9 / 61$ & 15 & 0.110 & $6 / 12$ & 50 \\
\hline Peritumoral lymphocytes & $5 / 18$ & 28 & $28 / 58$ & 48 & 0.175 & $8 / 12$ & 67 \\
\hline Intertumoral lymphocytes & $2 / 18$ & 11 & $24 / 61$ & 39 & 0.043 & $5 / 12$ & 42 \\
\hline Lymphangoinvasive growth & $7 / 18$ & 39 & $15 / 59$ & 25 & 0.371 & $2 / 12$ & 17 \\
\hline Lymph node metastasis & $10 / 18$ & 56 & $27 / 53$ & 51 & 0.790 & $2 / 12$ & 17 \\
\hline T-grade T3 \& T4 & $18 / 18$ & 100 & $41 / 60$ & 68 & 0.004 & $10 / 12$ & 83 \\
\hline
\end{tabular}

Bold $P$-values: $P$-values $<0.05$. 

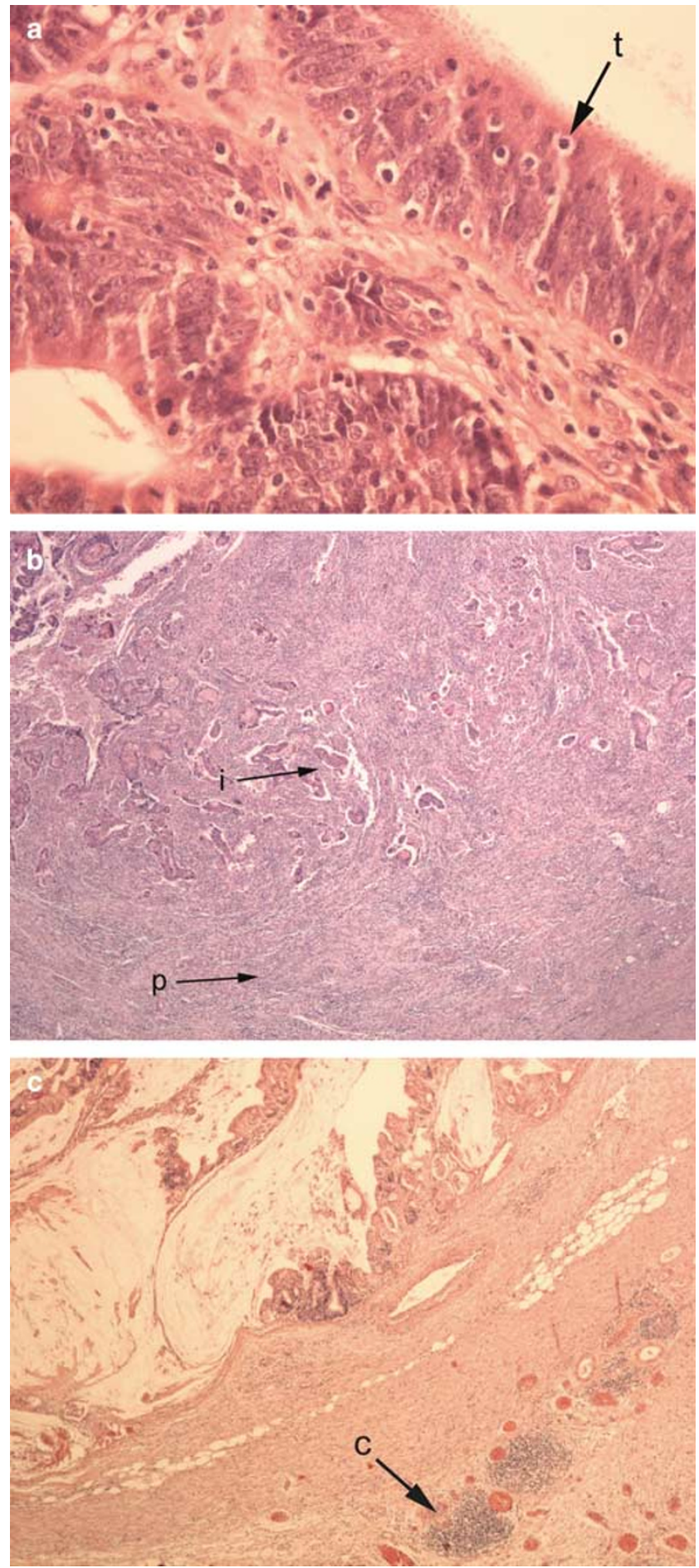

Figure 2 Morphology. (a) Intraepithelial (tumor infiltrating) lymphocytes $(\mathrm{t})$ in an MSI high tumor of patient with a pathogenic mutation in MLH1 (H\&E $\times 625)$. (b) inter (i)- and peri(p)tumoral lymphocytes in an MSS tumor $(\mathrm{H} \& \mathrm{E} \times 62.5)$. (c) Crohn's-like lymphoid reaction (c) in an MSI low tumor $(\mathrm{H} \& \mathrm{E} \times 35)$.

high group, underlines that there is no need to change the current practice in genetic counseling, which makes no difference in risk estimation for HNPCC between MSI low and MSS tumors.

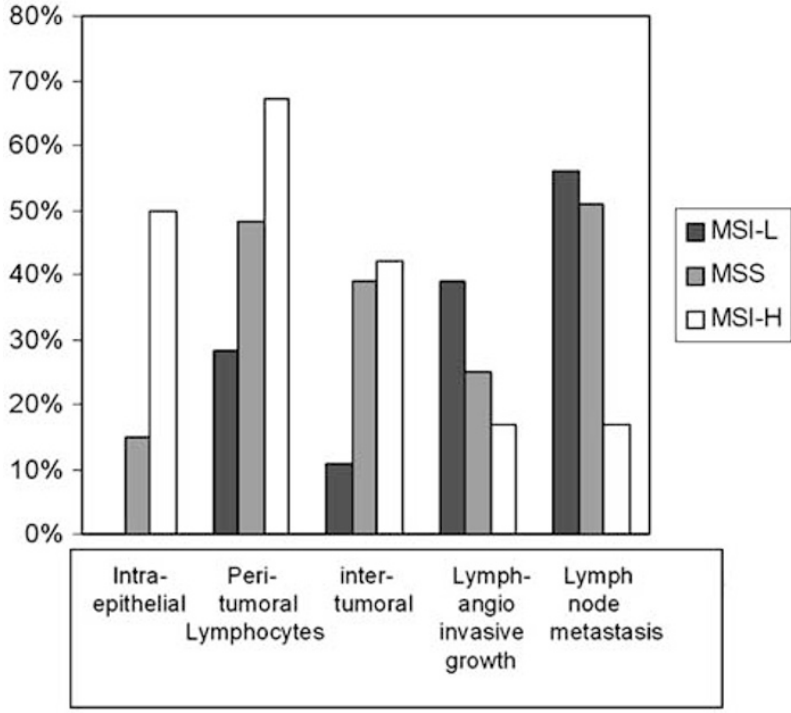

Figure 3 Presence of lymphocytes, lymph node metastasis and lymphangioinvasive growth.

In this study, the standard panel of five microsatellite markers ${ }^{1}$ is used to distinguish between MSI high, MSI low and MSS tumors. According to the guidelines a tumor is designated MSI low if only one of the five markers shows instability. The MSS tumors only included those in which all markers could unambiguously be characterized as stable. Despite of this, in the MSS group additional MSI low tumors will still be present, which would have become apparent when more markers would have been tested. ${ }^{19,20}$ Despite of the contamination of the MSS group with MSI low tumors, differences in pathological features between MSI low and MSS tumors were observed. This emphasizes that MSI low tumors form a separate entity within the group of familial CRCs.

MSI low tumors differ significantly from MSI high tumors both in the number of instable repeats and the degree of instability of a given microsatellite repeat, which is much less in MSI low tumors than in MSI high tumors. Like has been noticed before, ${ }^{21}$ in MSI low tumors the instability is predominantly limited to the addition of only one dinucleotide repeat, suggesting an underlying mechanism that is distinct from the MMR defects that are involved in HNPCC. It has been suggested that hypermethylation of the promoter of the $O^{6}$-methylguanine methyltransferase gene (MGMT) might be involved in the generation of MSI low tumors. ${ }^{5}$ However, this could not be confirmed by others ${ }^{22}$ and the etiology of MSI low tumors is not understood thus far.

In conclusion MSI low and MSS tumors are comparable with respect to family history, but MSI low tumors show unfavorable pathological characteristics as compared to MSS as well as MSI high cancers. This indicates a distinct underlying biology of MSI low CRC. 


\section{References}

1 Boland CR, Thibodeau SN, Hamilton SR, et al. A National Cancer Institute Workshop on microsatellite instability for cancer detection and familial predisposition: development of international criteria for the determination of microsatellite instability in colorectal cancer. Cancer Res 1998;58:5248-5257.

2 Tomlinson I, Halford S, Aaltonen L, et al. Does MSIlow exist? J Pathol 2002;197:6-13.

3 Kambara T, Matsubara N, Nakagawa H, et al. High frequency of low-level microsatellite instability in early colorectal cancer. Cancer Res 2001;61:7743-7746.

4 Jass JR, Biden KG, Cummings MC, et al. Characterisation of a subtype of colorectal cancer combining features of the suppressor and mild mutator pathways. J Clin Pathol 1999;52:455-460.

5 Whitehall VL, Walsh MD, Young J, et al. Methylation of O-6-methylguanine DNA methyltransferase characterizes a subset of colorectal cancer with low-level DNA microsatellite instability. Cancer Res 2001;61: 827-830.

6 Mori Y, Selaru FM, Sato F, et al. The impact of microsatellite instability on the molecular phenotype of colorectal tumors. Cancer Res 2003;63:4577-4582.

7 Kohonen-Corish MR, Daniel JJ, Chan C, et al. Low microsatellite instability is associated with poor prognosis in stage C colon cancer. J Clin Oncol 2005;23: 2318-2324.

8 Wright CM, Dent OF, Newland RC, et al. Low level microsatellite instability may be associated with reduced cancer specific survival in sporadic stage $C$ colorectal carcinoma. Gut 2005;54:103-108.

9 Rodriguez-Bigas MA, Boland CR, Hamilton SR, et al. A National Cancer Institute Workshop on hereditary nonpolyposis colorectal cancer syndrome: meeting highlights and Bethesda guidelines. J Natl Cancer Inst 1997;89:1758-1762.

10 American Gastroenterological Association (AGA). American Gastroenterological Association medical position statement: hereditary colorectal cancer and genetic testing. Gastroenterology 2001;121:195-197.

11 Hoogerbrugge N, Willems R, Van Krieken HJ, et al. Very low incidence of microsatellite instability in rectal cancers from families at risk for HNPCC. Clin Genet 2003;63:64-70.

12 Hamilton SR, Vogelstein B, Kudo S, et al. Tumours of the colon and rectum. In: Hamilton SR, Aaltonen LA (eds). World Health Organization Classification of Tumours, Pathology and Genetics, Tumours of the Digestive System. International Agency for Research on Cancer (IARC): Lyon (France), 2000, pp 103-119.

13 Graham DM, Appelman HD. Crohn's-like lymphoid reaction and colorectal carcinoma: a potential histologic prognosticator. Mod Pathol 1990;3:332-335.

14 van den Bos $M$, van den Hoven $M$, Jongejan E, et al. More differences between HNPCC-related and sporadic carcinomas from the endometrium as compared to the colon. Am J Surg Pathol 2004;28:706-711.

15 Pages F, Berger A, Camus M, et al. Effector memory T cells, early metastasis, and survival in colorectal cancer. N Engl J Med 2005;353:2654-2666.

16 Nagtegaal ID, Marijnen CA, Kranenbarg EK, et al. Local and distant recurrences in rectal cancer patients are predicted by the nonspecific immune response; specific immune response has only a systemic effect-a histopathological and immunohistochemical study. BMC Cancer 2001;1:7.

17 Jass JR, Do KA, Simms LA, et al. Morphology of sporadic colorectal cancer with DNA replication errors. Gut 1998;42:673-679.

18 Ward R, Meagher A, Tomlinson I, et al. Microsatellite instability and the clinicopathological features of sporadic colorectal cancer. Gut 2001;48:821-829.

19 Halford S, Sasieni P, Rowan A, et al. Low-level microsatellite instability occurs in most colorectal cancers and is a nonrandomly distributed quantitative trait. Cancer Res 2002;62:53-57.

20 Laiho P, Launonen V, Lahermo P, et al. Low-level microsatellite instability in most colorectal carcinomas. Cancer Res 2002;62:1166-1170.

21 Ikeda Y, Oda S, Abe T, et al. Features of microsatellite instability in colorectal cancer: comparison between colon and rectum. Oncology 2001;61:168-174.

22 Halford S, Rowan A, Sawyer E, et al. O(6)-methylguanine methyltransferase in colorectal cancers: detection of mutations, loss of expression, and weak association with G:C>A:T transitions. Gut 2005;54:797-802. 